\title{
ON THE VANISHING OF HOMOLOGY WITH MODULES OF FINITE LENGTH
}

\author{
PETTER ANDREAS BERGH
}

\begin{abstract}
We study the vanishing of homology and cohomology of a module of finite complete intersection dimension over a local ring. Given such a module of complexity $c$, we show that if $c$ (co)homology groups with a module of finite length vanish, then all higher (co)homology groups vanish.
\end{abstract}

\section{Introduction}

With his 1961 paper [1], Auslander initiated the study of vanishing of homology for modules over commutative Noetherian local rings. In that paper, he proved his famous rigidity theorem: if $M$ and $N$ are finitely generated modules over an unramified regular local ring $A$, then the implication

$$
\operatorname{Tor}_{n}^{A}(M, N)=0 \Longrightarrow \operatorname{Tor}_{i}^{A}(M, N)=0 \quad \text { for } \quad i \geq n
$$

holds for any $n \geq 0$. Lichtenbaum settled the ramified case in [10], and Murthy generalized this to arbitrary complete intersection rings in [11]. Namely, he proved that if $M$ and $N$ are finitely generated modules over a local complete intersection $A$ of codimension $c$, and

$$
\operatorname{Tor}_{n}^{A}(M, N)=\operatorname{Tor}_{n+1}^{A}(M, N)=\cdots=\operatorname{Tor}_{n+c}^{A}(M, N)=0
$$

for some $n \geq 0$, then $\operatorname{Tor}_{i}^{A}(M, N)=0$ for $i \geq n$. This was vastly generalized in [8], [9], where Jorgensen focused on the complexities of the modules involved, rather than the codimension of the ring. He showed that if $M$ is a module of finite complete intersection dimension and complexity $c$ over a local ring, then the vanishing of $c+1$ homology (respectively, cohomology) groups forces the vanishing of all the higher homology (respectively, cohomology) groups.

In all of the above mentioned vanishing results, one assumes the vanishing of consecutive (co)homology groups. However, the author showed in [4] that this is not necessary. In fact, the (co)homology groups assumed to vanish may be arbitrarily far apart from each other. Namely, let $M$ and $N$ be modules over a

Received 1 December 2009. 
local ring $A$, with $M$ of finite complete intersection dimension and complexity $c$. It was shown that if there exists an odd number $q$ such that

$$
\operatorname{Tor}_{n}^{A}(M, N)=\operatorname{Tor}_{n+q}^{A}(M, N)=\cdots=\operatorname{Tor}_{n+c q}^{A}(M, N)=0
$$

for some $n>\operatorname{depth} A-\operatorname{depth} M$, then $\operatorname{Tor}_{i}^{A}(M, N)=0$ for $i>\operatorname{depth} A-$ depth $M$ (and similarly for cohomology). The above vanishing result of Jorgensen is the special case $q=1$.

In this paper, we show that when the module $N$ has finite length, then we may reduce the number of (co)homology groups assumed to vanish by one. Namely, let $M$ and $N$ be modules over a local ring $A$, with $N$ of finite length and $M$ of finite complete intersection dimension and complexity $c$. In this situation, we show that if there exists an odd number $q$ such that

$$
\operatorname{Tor}_{n}^{A}(M, N)=\operatorname{Tor}_{n+q}^{A}(M, N)=\cdots=\operatorname{Tor}_{n+(c-1) q}^{A}(M, N)=0
$$

for some $n>\operatorname{depth} A-\operatorname{depth} M$, then $\operatorname{Tor}_{i}^{A}(M, N)=0$ for $i>\operatorname{depth} A-$ depth $M$ (and similarly for cohomology). The special case when $q=1$ and the ring is a complete intersection was proved by Jorgensen in the above mentioned papers.

\section{Complete intersection dimension}

Throughout this paper, we assume that all modules encountered are finitely generated. In this section, we fix a local (meaning commutative Noetherian local) ring $(A, \mathfrak{m}, k)$.

Every $A$-module $M$ admits a minimal free resolution

$$
\cdots \rightarrow F_{2} \rightarrow F_{1} \rightarrow F_{0} \rightarrow M \rightarrow 0
$$

which is unique up to isomorphism. The rank of the free $A$-module $F_{n}$ is the $n$th Betti number of $M$; we denote it by $\beta_{n}^{A}(M)$. The complexity of $M$, denoted cx $M$, is defined as

$$
\operatorname{cx} M \stackrel{\text { def }}{=} \inf \left\{t \in \mathrm{N} \cup\{0\} \mid \exists a \in \mathrm{R} \text { such that } \beta_{n}^{A}(M) \leq a n^{t-1} \text { for all } n \gg 0\right\} .
$$

Thus, the complexity of a module is the polynomial rate of growth of its Betti sequence.

An arbitrary local ring may have many modules with infinite complexity. In fact, by a theorem of Gulliksen (cf. [7, Theorem 2.3]), a local ring is a complete intersection if and only if all its modules have finite complexity. In order to study modules behaving homologically as modules over such rings, Avramov, Gasharov and Peeva defined in [2] the notion of modules with finite complete 
intersection dimension. Recall that a quasi-deformation of $A$ is a diagram $A \rightarrow R \leftarrow Q$ of local homomorphisms, in which $A \rightarrow R$ is faithfully flat, and $R \leftarrow Q$ is surjective with kernel generated by a regular sequence. The length of this regular sequence is the codimension of the quasi-deformation. An $A$-module $M$ has finite complete intersection dimension if there exists such a quasi-deformation in which the $Q$-module $R \otimes_{A} M$ has finite projective dimension. We shall write "CI-dimension" instead of "complete intersection dimension".

As mentioned, the homological behavior of modules of finite CI-dimension reflects the behavior of modules over local complete intersections. For example, such a module has finite complexity. Moreover, the cohomology groups are finitely generated over a ring of cohomology operators of degree two, a notion we now explain (cf. [2, 4.1]). Let $Q$ be a local ring, and let $x_{1}, \ldots, x_{c}$ be a regular sequence of length $c$. Denote the ring $Q /\left(x_{1}, \ldots, x_{c}\right)$ by $R$, and let $M$ be an $R$-module with a free resolution $\mathbf{F}$. The regular sequence gives rise to chain maps $\left\{t_{i} \in \operatorname{Hom}_{R}(\mathbf{F}, \mathbf{F})\right\}_{i=1}^{c}$ of degree -2, namely the Eisenbud operators. These are uniquely defined up to homotopy, and are therefore elements of $\operatorname{Ext}_{R}^{2}(M, M)$. Moreover, these chain maps commute up to homotopy. Thus, the Eisenbud operators give rise to a polynomial ring $R\left[\chi_{1}, \ldots, \chi_{c}\right]$ of operators with the following properties:

(1) The degree of $\chi_{i}$ is two for all $i$.

(2) There is a graded ring homomorphism $R\left[\chi_{1}, \ldots, \chi_{c}\right] \stackrel{\varphi_{M}}{\longrightarrow} \operatorname{Ext}_{R}^{*}(M, M)$ for every $R$-module $M$, where $\operatorname{Ext}_{R}^{*}(M, M)=\oplus_{n=0}^{\infty} \operatorname{Ext}_{R}^{n}(M, M)$.

(3) For every pair $(X, Y)$ of $R$-modules, the $R\left[\chi_{1}, \ldots, \chi_{c}\right]$-module structure on $\operatorname{Ext}_{R}^{*}(X, Y)$ through $\varphi_{X}$ and $\varphi_{Y}$ coincide (cf. [3, Theorem 3.3]).

(4) For every pair $(X, Y)$ of $R$-modules, the $R\left[\chi_{1}, \ldots, \chi_{c}\right]$-module $\operatorname{Ext}_{R}^{*}(X$, $Y$ ) is finitely generated whenever $\operatorname{Ext}_{Q}^{n}(X, Y)=0$ for $n \gg 0$ (cf. [6, Theorem 3.1]).

As mentioned above, modules of finite CI-dimension have finitely generated cohomology, as we see from property (4) above. Namely, let $M$ and $N$ be $A$ modules, with $M$ of finite CI-dimension over a quasi-deformation $A \rightarrow R \leftarrow$ $Q$, say. Furthermore, let $S$ be the polynomial ring induced by the Eisenbud operators coming from the surjection $Q \rightarrow R$. Then since $\operatorname{pd}_{Q}\left(R \otimes_{A} M\right)$ is finite, the $S$-module $\operatorname{Ext}_{R}^{*}\left(R \otimes_{A} M, R \otimes_{A} N\right)$ is finitely generated.

Having defined Eisenbud operators, we end this section with the following result. It shows that a module of finite CI-dimension and complexity one can be "realized" by a codimension one quasi-deformation.

Lemma 2.1. Let $A$ be a local ring, and let $M$ be an A-module of finite 
CI-dimension and complexity one. Then there exists a codimension one quasideformation $A \rightarrow R \leftarrow Q$ with $\operatorname{pd}_{Q}\left(R \otimes_{A} M\right)<\infty$.

Proof. By [2, Proposition 7.2(2)], there exists a quasi-deformation $A \rightarrow$ $R \leftarrow Q$ of codimension one, such that the Eisenbud operator $\chi$ on the minimal free resolution of $R \otimes_{A} M$ is eventually surjective. Thus, if

$$
\cdots \rightarrow F_{2} \rightarrow F_{1} \rightarrow F_{0} \rightarrow M \rightarrow 0
$$

is the minimal free resolution of $M$, and

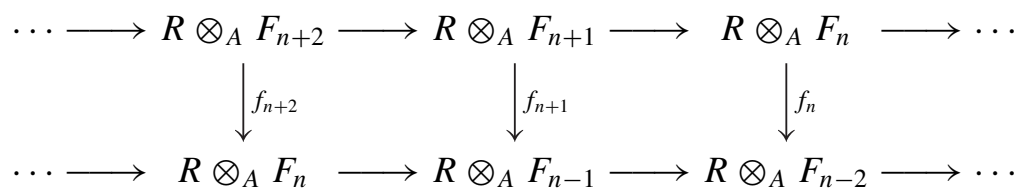

is the chain map corresponding to $\chi$, then $f_{n}$ is surjective for large $n$. By [2, Theorem 7.3], the minimal free resolution of $M$ is eventually periodic of period 2 , so that $F_{n}$ is isomorphic to $F_{n+2}$ for $n \gg 0$. Consequently, the map $f_{n}$ is an isomorphism for large $n$.

For any $R$-module $N$ and integer $n$, the action of $\chi$ on $\operatorname{Ext}_{R}^{n}\left(R \otimes_{A} M, N\right)$ is induced by $f_{n}$. Therefore, there exists an integer $n_{0}$ such that

$$
\chi \operatorname{Ext}_{R}^{n}\left(R \otimes_{A} M, N\right)=\operatorname{Ext}_{R}^{n+2}\left(R \otimes_{A} M, N\right)
$$

for all $n \geq n_{0}$. Consequently, the $R[\chi]$-module $\operatorname{Ext}_{R}^{n}\left(R \otimes_{A} M, N\right)$ is Noetherian, and so it follows from [2, Theorem 4.2] that $\operatorname{Ext}_{Q}^{n}\left(R \otimes_{A} M, N\right)$ vanishes for large $n$. In particular, this holds if we take $N$ to be the residue field of $Q$, hence $\operatorname{pd}_{Q}\left(R \otimes_{A} M\right)$ is finite.

\section{Vanishing of homology}

We start by showing the following special case of the main result. It shows that when a module has finite CI-dimension and complexity one, then the vanishing of a single homology group with a module of finite length implies the vanishing of all higher homology groups.

Proposition 3.1. Let $A$ be a local ring, and let $M$ be an A-module of finite CI-dimension and complexity one. Furthermore, let $N$ be an A-module of finite length. If $\operatorname{Tor}_{n}^{A}(M, N)=0$ for some $n>\operatorname{depth} A-\operatorname{depth} M$, then $\operatorname{Tor}_{n}^{A}(M, N)=0$ for all $n>\operatorname{depth} A-\operatorname{depth} M$.

Proof. Choose, by Lemma 2.1, a codimension one quasi-deformation $A \rightarrow R \leftarrow Q$ for which the projective dimension of the $Q$-module $R \otimes_{A} M$ is finite. Thus $R=Q /(x)$ for a regular element $x$ in $Q$. Denote the $R$-modules 
$R \otimes_{A} M$ and $R \otimes_{A} N$ by $M^{\prime}$ and $N^{\prime}$, respectively, and note that $N^{\prime}$ has finite length.

The change of rings spectral sequence

$$
\operatorname{Tor}_{p}^{R}\left(M^{\prime}, \operatorname{Tor}_{q}^{Q}\left(N^{\prime}, R\right)\right) \underset{p}{\Rightarrow} \operatorname{Tor}_{p+q}^{Q}\left(M^{\prime}, N^{\prime}\right)
$$

degenerates into a long exact sequence

$$
\begin{aligned}
& \operatorname{Tor}_{1}^{R}\left(M^{\prime}, N^{\prime}\right) \longrightarrow \operatorname{Tor}_{2}^{Q}\left(M^{\prime}, N^{\prime}\right) \longrightarrow \operatorname{Tor}_{2}^{R}\left(M^{\prime}, N^{\prime}\right) \longrightarrow \\
& \operatorname{Tor}_{0}^{R}\left(M^{\prime}, N^{\prime}\right) \longrightarrow \operatorname{Tor}_{1}^{Q}\left(M^{\prime}, N^{\prime}\right) \longrightarrow \operatorname{Tor}_{1}^{R}\left(M^{\prime}, N^{\prime}\right) \longrightarrow
\end{aligned}
$$

in homology. Let

$$
0 \rightarrow Q^{\beta_{d}^{Q}\left(M^{\prime}\right)} \rightarrow \cdots \rightarrow Q^{\beta_{0}^{Q}\left(M^{\prime}\right)} \rightarrow M^{\prime} \rightarrow 0
$$

be a minimal $Q$-free resolution of $M^{\prime}$. Localizing this resolution at $x$, keeping in mind that $x M^{\prime}=0$, we see that $\sum_{i=0}^{d}(-1)^{i} \beta_{i}^{Q}\left(M^{\prime}\right)=0$. Now $\ell\left(\operatorname{Tor}_{i}^{Q}\left(M^{\prime}\right.\right.$, $l))=\beta_{i}^{Q}\left(M^{\prime}\right)$, where $l$ is the residue field of $Q$. Moreover, the Euler characteristic $\sum_{i=0}^{\infty}(-1)^{i} \ell\left(\operatorname{Tor}_{i}^{Q}\left(M^{\prime},-\right)\right)$ is well defined and additive on the category of finite length $Q$-modules. Therefore, induction on length shows that $\sum_{i=0}^{\infty}(-1)^{i} \ell\left(\operatorname{Tor}_{i}^{Q}\left(M^{\prime}, X\right)\right)=0$ for every $Q$-module of finite length.

By the Auslander-Buchsbaum formula, the projective dimension of $M^{\prime}$ over $Q$ is given by

$$
\begin{aligned}
\operatorname{depth}_{Q} Q-\operatorname{depth}_{Q} M^{\prime} & =\operatorname{depth}_{R} R-\operatorname{depth}_{R} M^{\prime}+1 \\
& =\operatorname{depth}_{A} A-\operatorname{depth}_{A} M+1 .
\end{aligned}
$$

Let $m>\operatorname{depth}_{A} A-\operatorname{depth}_{A} M$ be an integer, and consider the long exact sequence in homology starting in $\operatorname{Tor}_{m+1}^{Q}\left(M^{\prime}, N^{\prime}\right)=0$, i.e. the long exact sequence obtained from the above spectral sequence. By taking the alternating sum of the lengths of the terms, and identifying $\operatorname{Tor}_{0}^{R}\left(M^{\prime}, N^{\prime}\right)$ with $\operatorname{Tor}_{0}^{Q}\left(M^{\prime}, N^{\prime}\right)$, we see that $\ell\left(\operatorname{Tor}_{m}^{R}\left(M^{\prime}, N^{\prime}\right)\right)=\ell\left(\operatorname{Tor}_{m+1}^{R}\left(M^{\prime}, N^{\prime}\right)\right)$.

Suppose that $\operatorname{Tor}_{n}^{A}(M, N)=0$ for some $n>\operatorname{depth} A-\operatorname{depth} M$. Since $\operatorname{Tor}_{n}^{R}\left(M^{\prime}, N^{\prime}\right)$ is isomorphic to $R \otimes_{A} \operatorname{Tor}_{n}^{A}(M, N)$, the above shows that $\operatorname{Tor}_{n}^{R}\left(M^{\prime}, N^{\prime}\right)=0$ for all $n>\operatorname{depth} A-\operatorname{depth} M$. Then by faithful flatness, the homology group $\operatorname{Tor}_{n}^{A}(M, N)$ vanishes for all $n>\operatorname{depth} A-\operatorname{depth} M$.

Having proved the complexity one case, we now prove the main result, the case when one of the modules has finite CI-dimension and complexity $c$, and the other has finite length. In this situation, the vanishing of $c$ homology 
groups implies the vanishing of all higher homology groups. Moreover, the "vanishing gap" can be any odd number. This result therefore generalizes [4, Theorem 3.5] to rings which are not necessarily complete intersections.

Theorem 3.2. Let $A$ be a local ring, and let $M$ be an A-module of finite CI-dimension and complexity $c$. Furthermore, let $N$ be an A-module of finite length. If there exist an odd number $q \geq 1$ and a number $n>\operatorname{depth} A-$ depth $M$ such that $\operatorname{Tor}_{i}^{A}(M, N)=0$ for $i \in\{n, n+q, \ldots, n+(c-1) q\}$, then $\operatorname{Tor}_{i}^{A}(M, N)=0$ for all $i>\operatorname{depth} A-\operatorname{depth} M$.

Proof. We argue by induction on the complexity $c$ of $M$. If $c=0$, then $\operatorname{pd} M=\operatorname{depth} A-\operatorname{depth} M$ by the Auslander-Buchsbaum formula, and the result follows. The case $c=1$ is Proposition 3.1, so we assume that $c \geq 2$.

By [5, Lemma 3.1], there exists a faithfully flat extension $A \rightarrow R$ of local rings with the following properties:

(1) There is an exact sequence

$$
0 \rightarrow R \otimes_{A} M \rightarrow K \rightarrow \Omega_{R}^{q}\left(R \otimes_{A} M\right) \rightarrow 0
$$

of $R$-modules.

(2) The $R$-modules $R \otimes_{A} M$ and $K$ have finite CI-dimension.

(3) The complexity of $K$ is $c-1$.

(4) $\operatorname{depth}_{R} R-\operatorname{depth}_{R} K=\operatorname{depth}_{A} A-\operatorname{depth}_{A} M$.

As in the previous proof, denote the $R$-modules $R \otimes_{A} M$ and $R \otimes_{A} N$ by $M^{\prime}$ and $N^{\prime}$, respectively, and note that $N^{\prime}$ has finite length. Since $\operatorname{Tor}_{i}^{R}\left(M^{\prime}, N^{\prime}\right)$ is isomorphic to $R \otimes_{A} \operatorname{Tor}_{i}^{A}(M, N)$ for all $i$, the vanishing assumption implies that $\operatorname{Tor}_{i}^{R}\left(M^{\prime}, N^{\prime}\right)=0$ for $i \in\{n, n+q, \ldots, n+(c-1) q\}$. From (1), we obtain a long exact sequence

$$
\begin{aligned}
\cdots \rightarrow \operatorname{Tor}_{i+q+1}^{R}\left(M^{\prime}, N^{\prime}\right) \rightarrow & \operatorname{Tor}_{i}^{R}\left(M^{\prime}, N^{\prime}\right) \\
& \rightarrow \operatorname{Tor}_{i}^{R}\left(K, N^{\prime}\right) \rightarrow \operatorname{Tor}_{i+q}^{R}\left(M^{\prime}, N^{\prime}\right) \rightarrow \cdots
\end{aligned}
$$

of homology groups, from which we obtain that $\operatorname{Tor}_{i}^{R}\left(K, N^{\prime}\right)=0$ for $i \in$ $\{n, n+q, \ldots, n+(c-2) q\}$. Therefore by induction and properties (2), (3) and (4), the homology group $\operatorname{Tor}_{i}^{R}\left(K, N^{\prime}\right)$ vanishes for all $i>\operatorname{depth}_{A} A-$ $\operatorname{depth}_{A} M$. The long exact sequence then shows that $\operatorname{Tor}_{i}^{R}\left(M^{\prime}, N^{\prime}\right) \simeq$ $\operatorname{Tor}_{i+j(q+1)}^{R}\left(M^{\prime}, N^{\prime}\right)$ for all $i>\operatorname{depth}_{A} A-\operatorname{depth}_{A} M$ and $j \geq 0$.

By considering the pairs $(i, j) \in\{(n, c),(n+q, c-1), \ldots,(n+(c-$ 1) $q, 1)\}$, we see that $\operatorname{Tor}_{i}^{R}\left(M^{\prime}, N^{\prime}\right)=0$ for $n+c q+1 \leq i \leq n+c q+c$. Faithful flatness then implies that $\operatorname{Tor}_{i}^{A}(M, N)=0$ for $n+c q+1 \leq i \leq n+c q+c$. Thus we have reduced to the case when $c$ consecutive homology groups vanish. 
Now we use [5, Lemma 3.1] once more: there exists a faithfully flat local homomorphism $A \rightarrow S$ and an exact sequence

$$
0 \rightarrow S \otimes_{A} M \rightarrow K^{\prime} \rightarrow \Omega_{S}^{1}\left(S \otimes_{A} M\right) \rightarrow 0
$$

of $S$ modules, in which $S \otimes_{A} M$ and $K^{\prime}$ satisfy properties (2), (3) and (4). Arguing as above, we obtain an isomorphism

$$
\operatorname{Tor}_{i}^{S}\left(S \otimes_{A} M, S \otimes_{A} N\right) \simeq \operatorname{Tor}_{i+2}^{S}\left(S \otimes_{A} M, S \otimes_{A} N\right)
$$

for all $i>\operatorname{depth}_{A} A-\operatorname{depth}_{A} M$. Since $\operatorname{Tor}_{i}^{S}\left(S \otimes_{A} M, S \otimes_{A} N\right)=0$ for $n+c q+1 \leq i \leq n+c q+c$ and $c$ is at least two, we conclude that $\operatorname{Tor}_{i}^{S}\left(S \otimes_{A} M, S \otimes_{A} N\right)=0$ for all $i>\operatorname{depth}_{A} A-\operatorname{depth}_{A} M$. Finally, faithful flatness implies that $\operatorname{Tor}_{i}^{A}(M, N)=0$ for all $i>\operatorname{depth}_{A} A-\operatorname{depth}_{A} M$.

We record the special case $q=1$ in the following corollary, i.e. the case when $c$ consecutive homology groups vanish. Note that the case when the ring is a complete intersection follows from [8, Theorem 2.6].

Corollary 3.3. Let $A$ be a local ring, and let $M$ be an A-module of finite CI-dimension and complexity $c$. Furthermore, let $N$ be an A-module of finite length. If there exists a number $n>\operatorname{depth} A-\operatorname{depth} M$ such that $\operatorname{Tor}_{i}^{A}(M, N)=0$ for $n \leq i \leq n+c-1$, then $\operatorname{Tor}_{i}^{A}(M, N)=0$ for all $i>$ depth $A-\operatorname{depth} M$.

We also include the cohomology versions of Theorem 3.2 and Corollary 3.3. We do not include a proof; the proofs of Proposition 3.1 and Theorem 3.2 carry over verbatim to the cohomology case.

Theorem 3.4. Let $A$ be a local ring, and let $M$ be an A-module of finite CI-dimension and complexity $c$. Furthermore, let $N$ be an A-module of finite length. If there exist an odd number $q \geq 1$ and a number $n>\operatorname{depth} A-$ depth $M$ such that $\operatorname{Ext}_{A}^{i}(M, N)=0$ for $i \in\{n, n+q, \ldots, n+(c-1) q\}$, then $\operatorname{Ext}_{A}^{i}(M, N)=0$ for all $i>\operatorname{depth} A-\operatorname{depth} M$.

Corollary 3.5. Let $A$ be a local ring, and let $M$ be an A-module of finite CI-dimension and complexity c. Furthermore, let $N$ be an A-module of finite length. If there exists a number $n>\operatorname{depth} A-\operatorname{depth} M$ such that $\operatorname{Ext}_{A}^{i}(M, N)=0$ for $n \leq i \leq n+c-1$, then $\operatorname{Ext}_{A}^{i}(M, N)=0$ for all $i>\operatorname{depth} A-\operatorname{depth} M$. 


\section{REFERENCES}

1. Auslander,M., Modules over unramified regular local rings, Illinois. J. Math. 5 (1961), 631647.

2. Avramov, L., Gasharov, V., Peeva, I., Complete intersection dimension, Inst. Hautes Études Sci. Publ. Math. 86 (1997), 67-114.

3. Avramov, L., Sun, L.-C., Cohomology operators defined by a deformation, J. Algebra 204 (1998), 684-710.

4. Bergh,P. A., On the vanishing of (co)homology over local rings, J. Pure Appl. Algebra 212 (2008), 262-270.

5. Bergh,P. A., Jorgensen, D., On the vanishing of homology for modules of finite complete intersection dimension, J. Pure Appl. Algebra 215 (2011), 242-252.

6. Gulliksen, T. H., A change of ring theorem with applications to Poincaré series and intersection multiplicity, Math. Scand. 34 (1974), 167-183.

7. Gulliksen, T. H., On the deviations of a local ring, Math. Scand. 47 (1980), 5-20.

8. Jorgensen, D., Complexity and Tor on a complete intersection, J. Algebra 211 (1999), 578598.

9. Jorgensen, D. Vanishing of (co)homology over commutative rings, Comm. Alg. 29 (2001), 1883-1898.

10. Lichtenbaum, S., On the vanishing of Tor in regular local rings, Illinois J. Math. 10 (1966), 220-226.

11. Murthy, M., Modules over regular local rings, Illinois J. Math. 7 (1963), 558-565.

INSTITUTT FOR MATEMATISKE FAG

NTNU

N-7491 TRONDHEIM

NORWAY

E-mail: bergh@math.ntnu.no 